\title{
Un estudio de caso: Rehabilitación singular de edificios de viviendas en la barriada del Parque Alcosa, análisis de daños constructivos comunes y propuesta de intervención
}

\section{A case study: the rehabilitation of residential buildings in Parque Alcosa district, analysis of common diseases and intervention proposal}

$\underline{\text { R. D. Cano-Marín }}^{(*)}$, A. Jaramillo-Morilla ${ }^{(*)}$, F.J. Bernal-Serrano ${ }^{(*)}$, D. Moreno-Rangel ${ }^{(*)}$

\section{RESUMEN}

La barriada del Parque Alcosa se localiza en el noroeste del núcleo urbano de Sevilla, y está formada por un conjunto de 10.640 viviendas de p romoción pública construida durante los años 69-72 por el constructor valenciano Alfredo Corral. Existen tres modelos de edificación diferentes que responden a fases de construcción, siendo objeto de estudio en este artículo la correspondiente a la primera fase, comprendidas por las calles Ciudad de Játiva, Gandía, Sueca, Onteniente, Carcagente, Burjasot, Godella, Alfafar, Buñol, Paterna y Oliva. El presente trabajo expone el análisis de las patologías constructivas comunes existentes en la fase 1 del Parque Alcosa, relacionadas con el carácter potencialmente expansivo de los terrenos donde se ubica. Así mismo se desarrollan las soluciones constructivas de la intervención proyectada, llevado a cabo mediante el programa de Rehabilitación Singular de Edificios de la Empresa Pública del Suelo de Andalucía.

Palabras clave: Rehabilitación de edificios; patología constructiva; asiento diferencial; arcillas expansivas.

\section{ABSTRACT}

The Parque Alcosa district is located in northwestern area of Seville. It consists of 10,640 public housing development, that was promoted throughout the years 69-72 by the builder Alfredo Corral. There are three different building types which correspond to the different building stages. This article focuses on the the first one, which includes the streets, Ciudad de Jativa, Gandía, Sueca, Onteniente, Carcagente, Burjasot, Godella, Alfafar, Buñol, Paterna y Oliva. The present paper provides a constructive analisys of common building pathologies in phase 1 of Parque Alcosa, related to the potentially expansive features of the land where it is located. This paper also describes the structural sollutions for the projected intervention designed by the architect who subscribes, and was supported by the Public Land Company of Andalusia, under the Singular Building Rehabilitation program.

Keywords: Building rehabilitation; building pathology; differential movement; expansive clais.

(*) ETSA - Universidad de Sevilla (España).

Persona de contacto/Corresponding Author: rubendariocano@hotmail.com (R.D. Cano)

Cómo citar este artículo/Citation: Cano-Marín, R.D., Jaramillo-Morilla, A., Bernal-Serrano, F.J., Moreno-Rangel, D. (2014). Un estudio de caso: Rehabilitación singular de edificios de viviendas en la barriada del Parque Alcosa, análisis de daños constructivos comunes y propuesta de intervención. Informes de la Construcción, 66(534): e017, doi: http://dx.doi.org/10.3989/ic.12.112.

Licencia/License: Salvo indicación contraria, todos los contenidos de la edición electrónica de Informes de la Construcción se distribuyen bajo una licencia de uso y distribución Creative Commons Reconocimiento no Comercial 3.o. España (cc-by-nc). 


\section{OBJETIVO DEL ESTUDIO}

Tras la puesta en marcha del plan de rehabilitación singular de edificios llevado a cabo por la Empresa Pública del Suelo de Andalucía en los años 2006 y 2007, se elaboran los proyectos de rehabilitación de la zona del Parque Alcosa correspondiente a la Fase ${ }^{\mathrm{a}}$, reflejada en la Figura 1.

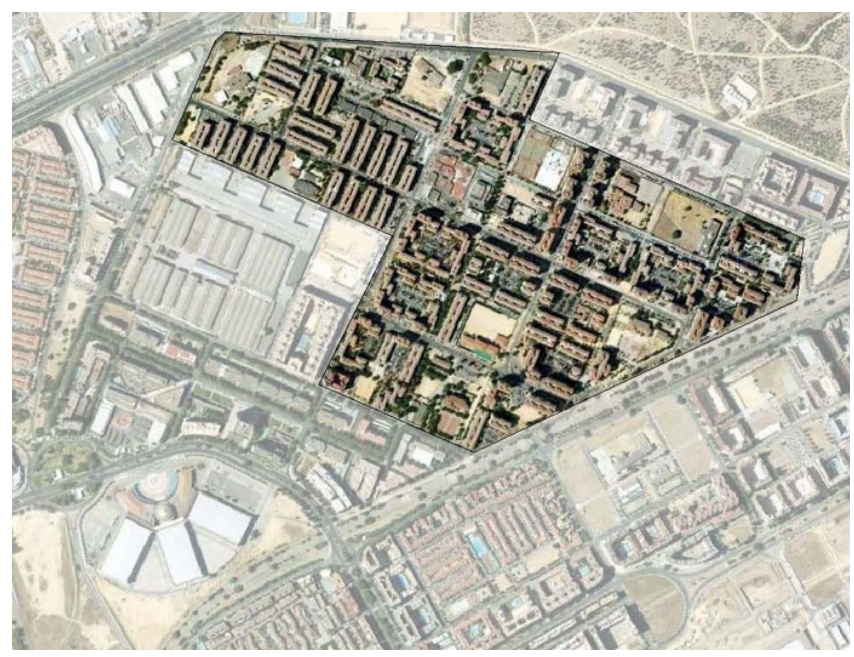

Figura 1. Barriada del Parque Alcosa

El programa de rehabilitación singular de edificios se basa en la redacción de proyectos que contemplan la intervención individual en comunidades de propietarios, en este caso integradas en manzanas compuestas por 6 comunidades, que comparten el mismo sistema constructivo (cimentación, estructura, sistema de cubierta y patios intercomunitarios) y, por tanto los mismas problemas constructivos.

Tras la elaboración de un estudio de viabilidad para el análisis de los daños constructivos existentes en los edificios de la zona, se percibe la existencia de forma sistemática de dos problemas comunes en todas las comunidades visitadas:

1. Humedad capilar en paramentos y muros de carga de zonas comunes y privativas de la planta baja.

2. Grietas en las cajas de escaleras de los edificios, que en algún caso puntual podía llegar a provocar el descalce de la zanca de escalera en las plantas superiores.

El presente artículo tiene el objetivo de analizar las causas de los daños descritos, exponer la metodología de trabajo desarrollada para su seguimiento, así como plantear los fundamentos para una intervención generalizada en la zona, que cumpliendo con los requisitos del programa de Rehabilitación al que se acogen las obras, pudieran ser llevadas a cabo de forma individual por cada comunidad de propietarios, buscando de este modo el equilibrio entre idoneidad técnica y sostenibilidad económica de las obras.

\section{FUENTES DE LA INFORMACIÓN}

La información recopilada para la confección de este artículo proviene en su mayor parte de la obtenida a raíz de la redacción de los proyectos de rehabilitación de los edificios y las direcciones de obras encargadas por la Empresa Pública del Suelo de Andalucía, así como de la consulta de los proyectos originales de estos edificios, documentación archivada en los depósitos de la Fundación para la Investigación de la Arquitectura.

\section{CONTEXTO SOCIAL Y URBANO}

Bajo el nombre de «Parque Alcosa» se encierra un barrio originalmente aislado situado al noreste del núcleo urbano Sevilla, junto a la autovía A-4, a medio camino entre el centro urbano y el aeropuerto de San Pablo.

Los criterios racionalistas que inspiraron el diseño del barrio suponen una estructura viaria de calles anchas, zonas ajardinadas con vegetación de gran porte y espacios parcialmente dedicados a aparcamiento.

Tiene su origen en una promoción privada de viviendas baratas en bloque desarrollada entre 1969 y 1972. Su alejamiento del centro y la falta de previsión en los planes urbanísticos tuvieron como consecuencia la carencia de equipamientos e infraestructuras básicas de saneamiento y abastecimiento de agua, problemas que posteriormente fueron solventados tras la redacción de los Estudios de Detalle en la zona.

El Plan General de Ordenación Urbana de Sevilla de 2006 clasifica el ámbito en la zona de ordenanza relativa a edificación abierta de edificios o polígonos de vivienda plurifamiliar en altura, generalmente en bloques aislados, estableciendo las siguientes figuras de planeamiento que afectan al área de intervención:

- ARI-DE-01 Virgen de los Reyes, que prevé destinar un antiguo polígono industrial al suroeste del barrio a usos residenciales, equipamientos y espacios libres.

- API-DE-06 Parque Alcosa, que incorpora el Estudio de Detalle UA-AE-1 del Plan General de 1987, modificado en 1998, para el desarrollo residencial de la franja norte del barrio.

\section{METODOLOGÍA}

La metodología aplicada para el análisis y la evaluación de los edificios se puede sintetizar en las fases y actuaciones siguientes:

Una fase inicial de recopilación de información previa sobre los edificios, a partir de los proyectos originales facilitados por la Fundación de Investigación de la Arquitectura, y de las características del terreno, datos obtenidos por medio del Instituto Geológico y Minero de España, el Protocolo de Inspección Técnica de Edificios, (1) y estudios geotécnicos realizados en la zona.

Una segunda fase de recopilación de información in situ, programando un plan de visitas a los edificios, zonas exteriores ajardinadas, zonas comunitarias y privativas, con el fin de evaluar sus daños aparentes y estado de conservación. Esta visita se llevó a cabo en 12 comunidades de propietarios, ubicadas en las calles Ciudad de Onteniente 2, 4, 5, 6, Ciudad de Cullera 2 y 7, Ciudad de Játiva 2, Ciudad de Gandía 5, Ciudad de Sueca 5, Ciudad de Ayora 3, Ciudad de Liria 2, Ciudad de Carlet 10.

Una tercera fase consistente en la toma de datos y que podríamos relacionar como sigue: 
- Levantamiento de planos.

- Reconocimiento de los forjados sanitarios.

- Levantamiento de daños por humedad capilar, de condensación o de filtración.

- Levantamiento de daños por grietas.

- Nivelación topográfica de la comunidad de propietarios con mayor afección por grietas, situada en C/ Ciudad de Onteniente 4 .

\section{1. Ámbitos temáticos de análisis}

Mediante la información obtenida con el estudio y la toma de datos definida anteriormente elaboramos un análisis compuesto por los siguientes ámbitos temáticos, del que se han deducido posteriormente el estudio de causas y las propuestas de intervención.

- Análisis constructivo del edificio.

- Análisis del terreno.

- Análisis del entorno.

4.1.1. Análisis constructivo

La fase 1 del Parque Alcosa está formada por bloques de 6 comunidades de propietarios en manzana con patios interiores, de dimensiones 76,00 $\mathrm{m}$ de largo $\times 22,58 \mathrm{~m}$ de ancho.

El bloque aislado está formada por dos modelos de edificación reflejados en la Figura 2 como A y B. El bloque tipo A se ubica en los extremos de la manzana, consta de dos viviendas por planta de $74 \mathrm{~m}^{2}$ construidos y está constituido estructuralmente por dos crujías paralelas a vial. El tipo B se sitúa en el interior de la manzana, sumando un total de 4 bloques en $\mathrm{H}$ con 4 viviendas por planta de $76 \mathrm{~m}^{2}$, y está definido por cuatro crujías, dos paralelas a la fachada delantera y dos a la fachada trasera.

En total cada manzana consta de una superficie construida de $11.856 \mathrm{~m}^{2}$, en una parcela de $1.976 \mathrm{~m}^{2}$, donde se ubican un total de 120 viviendas.

Los edificios que componen las manzanas comparten una cimentación ejecutada mediante un forjado sanitario con altura libre de $80 \mathrm{~cm}$, formado por jácenas hormigón armado de $70 \mathrm{~cm}$ canto sobre las que apoyan los muros de carga. Embrochalando estas jácenas se disponen una vigas de atado de hormigón armado de $40 \mathrm{~cm}$ de canto, y en el encuentro de jácenas y vigas de atado unos pozos de cimentación troncocónicos de hormigón en masa de 2,20 m de profundidad con una base de $2 \mathrm{~m}$ de diámetro, según el detalle de la Figura 3.

La estructura se ejecuta mediante muros de carga de bloques de hormigón vibrado de $20 \mathrm{~cm}$ de espesor paralelos a vial, en crujías de 3,55 m de ancho. Sobre los muros se disponen vigas de $40 \mathrm{~cm}$ de canto y nervios de borde perpendiculares a fachada, que en el caso de las cajas de escalera se resuelven mediante piezas en «U» de hormigón prefabricado como encofrado de perdido. Los forjados son en todos los casos unidireccionales de viguetas pretensadas.

Los patios interiores carecen de cimentación, haciéndose uso de una solera apoyada directamente en un relleno compactado de tierras procedentes de las propias obras de excavación del edificio.

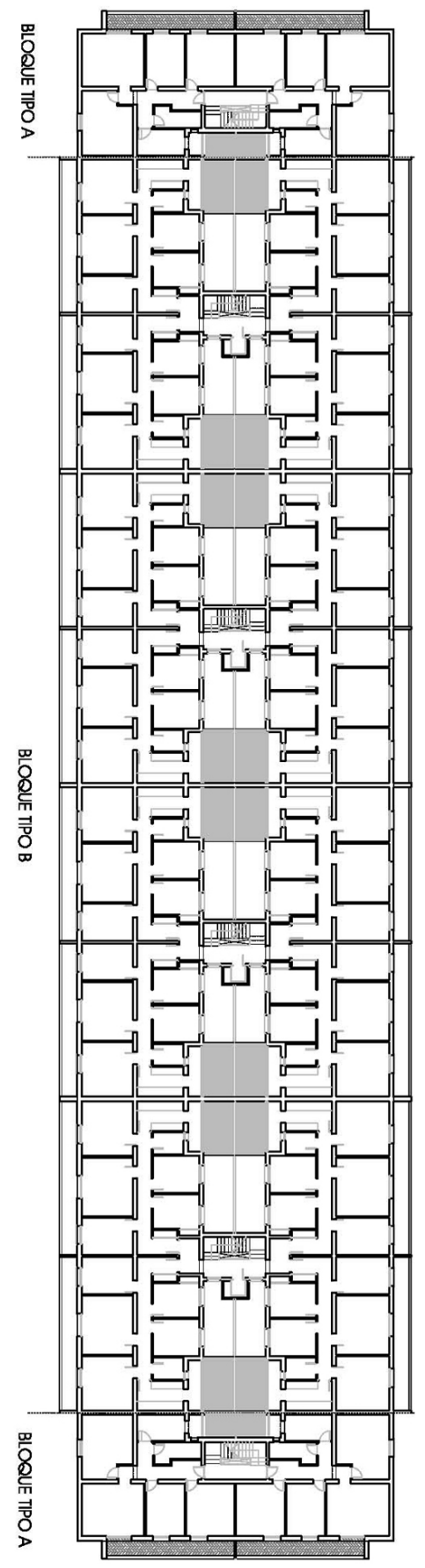

Figura 2. Esquema planta tipo de manzana

La instalación de saneamiento horizontal, ejecutada mediante arquetas de fábrica de ladillo y colectores de fibrocemento, parte de los patios interiores quedando embebidas en el terreno y no resultando registrable hasta su encuentro con el trazado ubicado en el vestíbulo del edificio, donde las arquetas tienen su base apoyada directamente en el terreno y son accesibles desde el forjado sanitario, según el detalle de la Figura 4.

Las cubiertas se resuelven inclinadas sobre tabiques palomeros terminadas en teja cerámica plana, y plana no transitable en la superficie correspondiente a la caja de escalera y pasillo distribuidor.

Las escaleras se ejecutan mediante bóveda tabicada mediante rosca de ladrillo macizo empotrada en los muros de carga. 


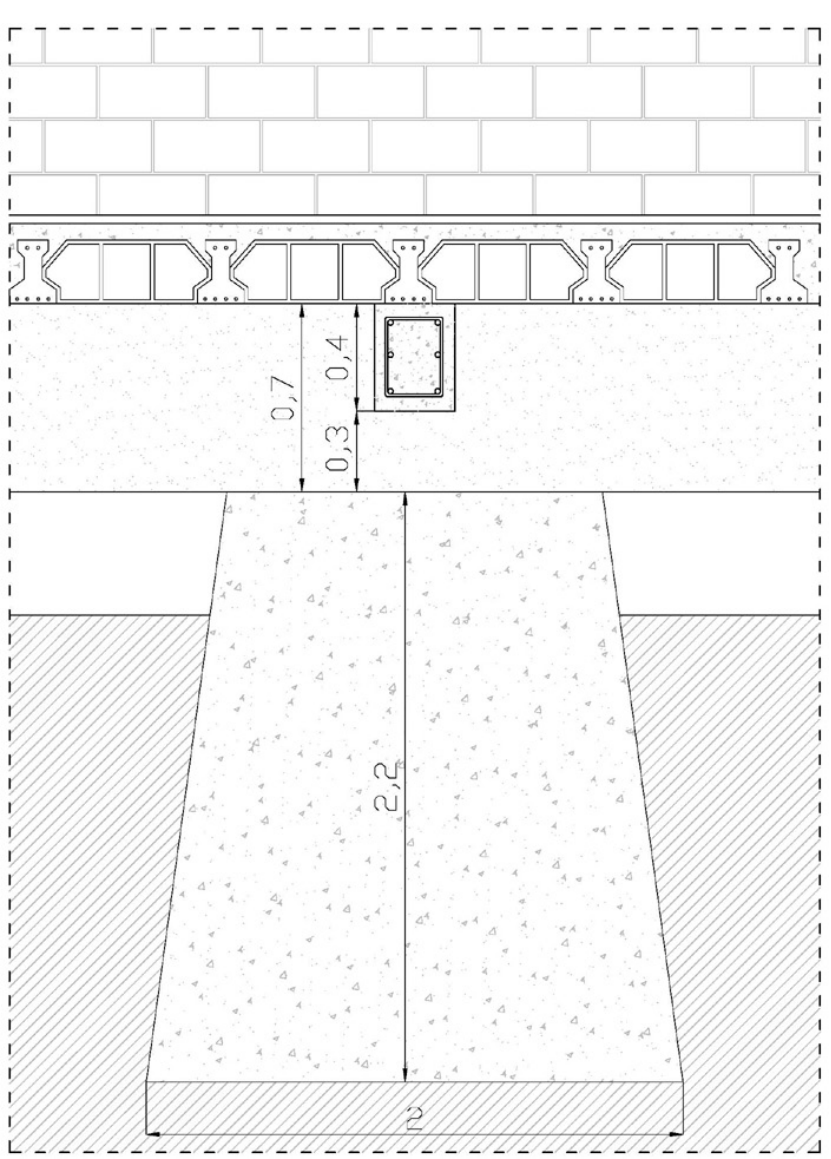

Figura 3. Detalle de cimentación

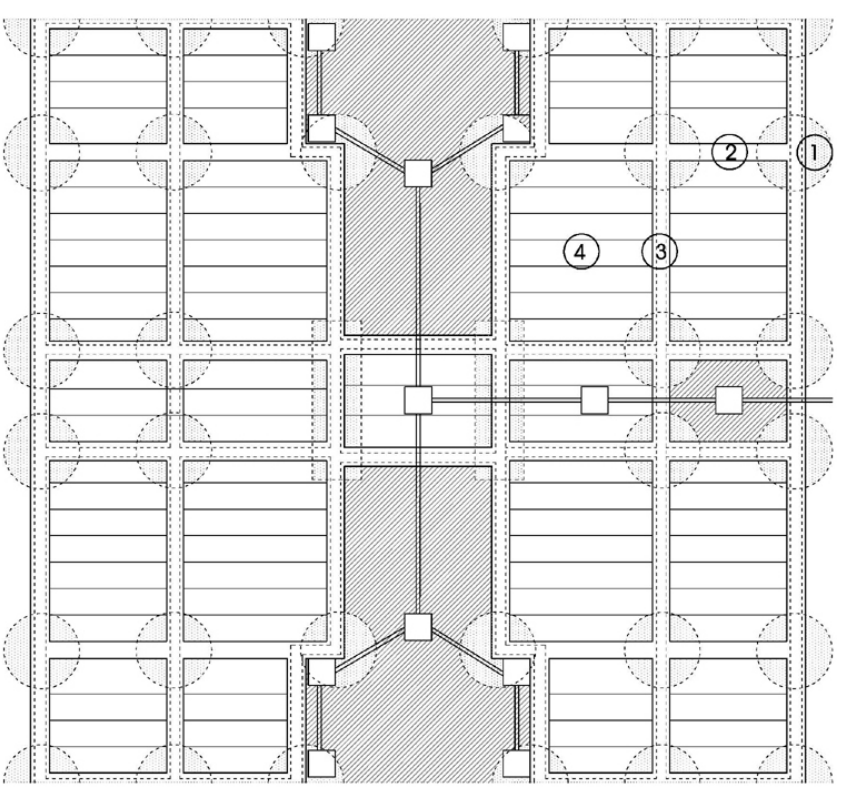

Figura 4. Esquema en planta de la cimentación y sección del edificio, con la siguiente correspondencia: 1 pozo de cimentación, 2 vigas de atado, 3 jácenas y muro de carga y 4 forjado sanitario.

\subsubsection{Análisis del terreno}

Para el análisis de las características del terreno se recurre al estudio de la siguiente documentación:
Mapa Geotécnico Básico de la Ciudad de Sevilla, anejo 1.2 del Protocolo de Inspección Técnica de Edificios, del que extraemos que el techo de la capa de las arcillas se ubica a -0,5 $\mathrm{m}$ y su espesor varía en la zona de actuación entre 5 y $8 \mathrm{~m}$.

Mapa Geológico de España hoja 984/12-40 (2), obtenido del IGME, Instituto Geológico y Minero de España, del que se desprende que la Barriada de Alcosa se asienta sobre un "suelo cuaternario de edad andaluciense denominado QT2 formado por cantos rodados y arcillas».

Mapa Geológico General, Mapa de Interpretación Geotécnica página 75/3-10, del que se desprende que la Barriada de Alcosa se sitúa en la zona II1 denominada «Recintos hundidos de la Depresión del Guadalquivir» con las siguientes características:

«Gravas, arenas, arcillas y limos... predominantemente permeable, existen acuíferos por porosidad intergranular. Nivel freático elevado... capacidad de carga baja con asientos de magnitud media que pueden ser importantes en zonas limo arcillosas...»

Estudio geotécnico para el «Reconocimiento Geotécnico para centro de Salud en Parque Alcosa», realizado por Geotécnica y Control de Calidad en 1987, del que extraemos los siguientes datos:

«Capa 1: arcilla marrón rojiza oscura, potencia $1 \mathrm{~m}$., Capa 2: arcilla marrón rojiza y marrón con nódulos y vetas calcáreas. Potencia 3,9 m; Capa 3: Gravas con matriz arcillo arenosa, a partir de 4,9 m. Presión de hinchamiento 1,5 Kp/ $\mathrm{cm}^{2}$. Expansividad media - alta y nivel freático - 3,35 $\mathrm{m»}$.

Por la documentación analizada comprobamos que se trata de un terreno arcilloso cuya capa activa alcanza aproximadamente 4 metros de profundidad y cuya presión de hinchamiento se aproxima a $1,5 \mathrm{Kp} / \mathrm{cm}^{2}$, lo que corresponde a un suelo con grado de expansividad elevado ${ }^{1}$.

En base a las cargas empleadas por normativa comprobamos que los pozos de cimentación transmiten una carga aproximada al terreno de $2,55 \mathrm{Kp} / \mathrm{cm}^{2}$ en el caso de las crujías intermedias, y de $1,85 \mathrm{Kp} / \mathrm{cm}^{2}$ en el caso de las ubicadas en fachadas exteriores e interiores, carga superior a la presión de hinchamiento de las arcillas.

\subsubsection{Entorno}

Las manzanas orientan su fachada delantera hacia una bolsa de aparcamientos con acceso desde la avenida principal Ciudad de Chivas, y la fachada trasera hacia una zona ajardinada intercomunitaria.

Todas las zonas ajardinadas constan de árboles de gran envergadura, siendo un ejemplo común el Celtis Australis o almez, que puede llegar a alcanzar los $25 \mathrm{~m}$ de altura.

Estos árboles se sitúan a distancias inferiores a 6 metros de las fachadas traseras, con lo que las raíces interfieren en el ámbito de las cimentaciones de los edificios.

\footnotetext{
${ }^{1}$ La guía de previsión de riesgos por expansividad de arcillas (3), establece el potencial y el grado de expansividad de las arcillas en base a la presión de hinchamiento y el hinchamiento libre máximo.
} 


\section{ANALISIS DE LOS DAÑOS COMUNES EXISTENTES}

Realizada la inspección de las 12 comunidades antes relacionadas, comprobamos la existencia de dos patologías generalizadas.

\subsection{Grietas en cajas de escalera}

Surgen en el encuentro de las zancas, forjado y cerramiento de las cajas de escalera, con los muros de carga correspondientes a las fachadas interiores del edificio.

Estas grietas prácticamente son inapreciables entre la planta baja y la segunda, y aumentan progresivamente de espesor llegando a alcanzar $8 \mathrm{~mm}$ en la última planta, según refleja las Figuras 5 y 6.

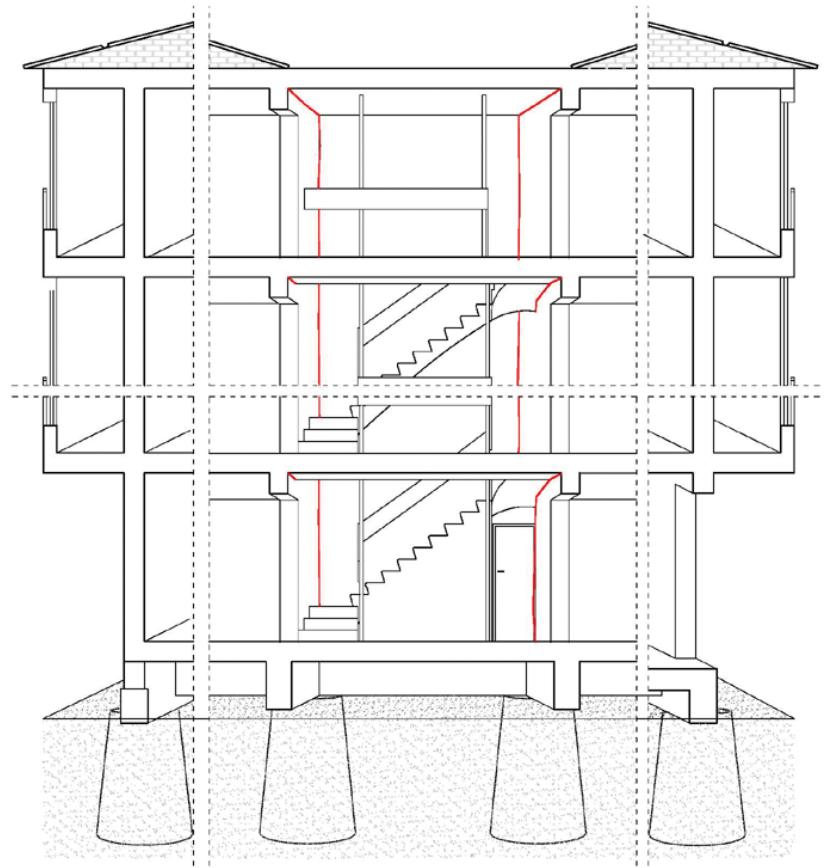

Figura 5. Esquema gráfico de las grietas

\subsection{Humedad capilar en suelos y paramentos de planta baja}

Comprobamos la existencia de humedad capilar en muros de carga y cerramientos de la planta baja del edificio, así como en la tabiquería interior y solería de las viviendas, dando lugar al oscurecimiento de las llagas y la pudrición de la carpintería de madera, marcos y puertas de paso, como puede apreciarse en la Figura 7.

Estos daños por humedad surgen en la planta baja de forma generalizada, aunque se manifiestan con mayor intensidad en las fachadas interiores y exteriores del edificio, tal como puede extraerse de la Figura 8, levantamiento de daños del edificio sito en $\mathrm{C} /$ Ciudad de Onteniente 4.

\section{ANÁLISIS DE CAUSAS}

\subsection{Causa de los daños por filtraciones}

Del reconocimiento de los forjados sanitarios se extrae que de forma generalizada se produce el embalsamiento de agua,

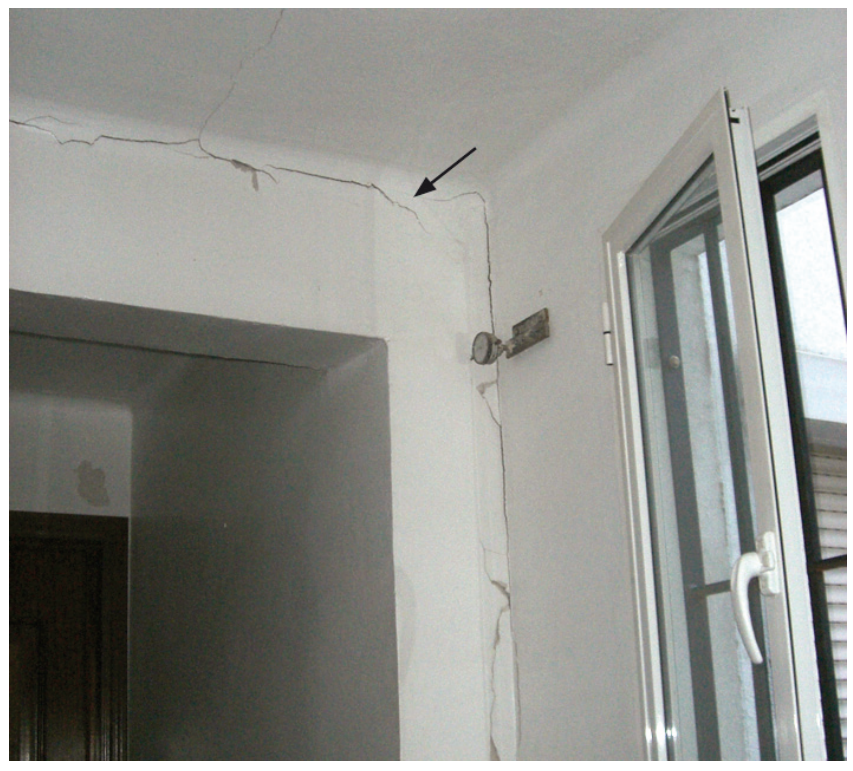

Figura 6. Grietas en caja de escalera C/ Ciudad de Onteniente 4.

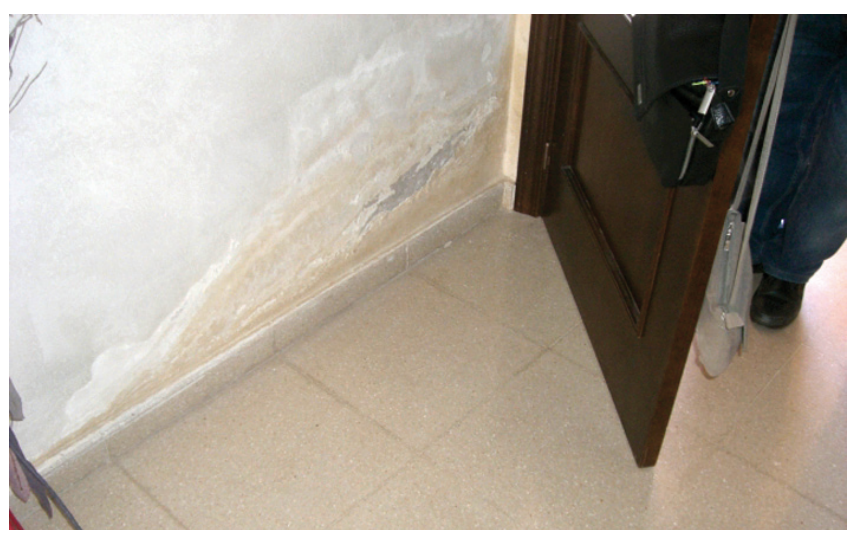

Figura 7. Humedad capilar en vivienda de planta baja.

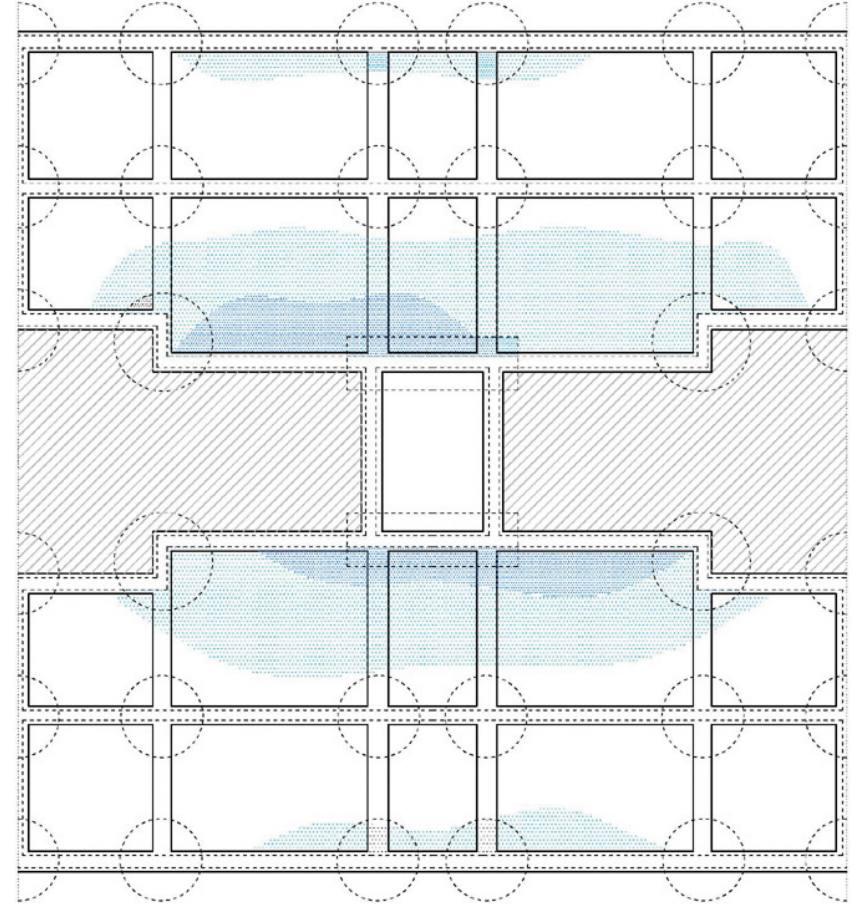

Figura 8. Humedad capilar en planta baja del edificio sito en C/ Ciudad de Onteniente 4. 
según refleja la Figura 9, debido a una filtración que puede tener como origen las siguientes causas:

- Filtraciones desde las redes de saneamiento horizontal de la comunidad de propietarios o de las comunidades colindantes.

- Filtración por el retroceso de agua desde la red de alcantarillado público en época de fuertes lluvias debido a la falta de dimensionamiento y mantenimiento.

- Filtración de agua pluvial desde el encuentro del acerado con la fachada delantera y trasera.

- Filtración de agua pluvial o de riego filtrada al terreno desde las zonas ajardinadas anexas a las manzanas.

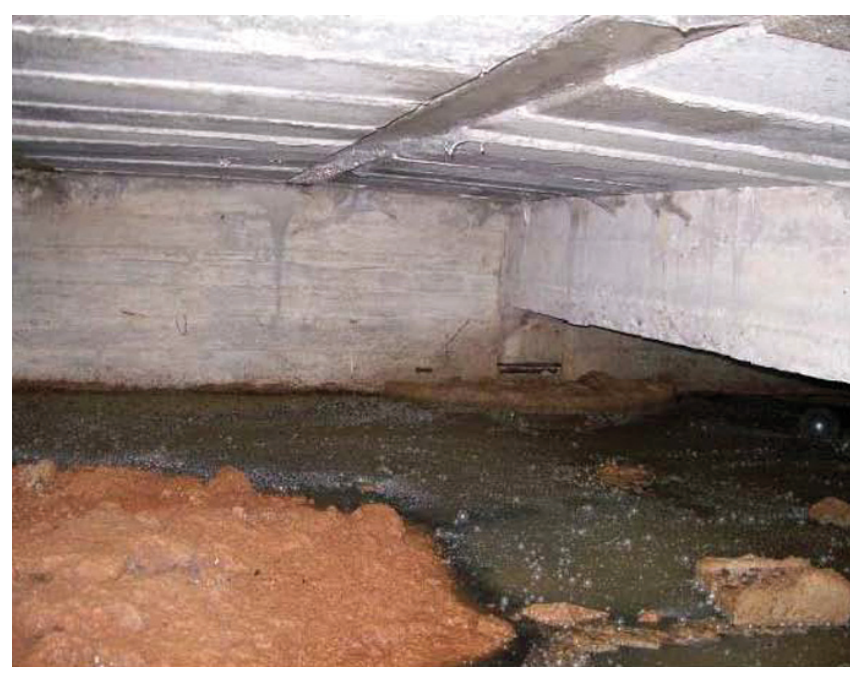

Figura 9. Embalsamiento de agua en la cámara bajo el forjado sanitario.

\subsubsection{Filtraciones desde las redes de saneamiento horizontal}

Las redes de saneamiento, ejecutadas mediante arquetas de fábrica de ladrillo y colectores de fibrocemento apoyados en el terreno, cuentan con uniones rígidas y frágiles incapaces de asumir las deformaciones inducidas por el suelo expansivo sin provocar fugas y filtraciones de agua al terreno.

Dado que el relleno de tierras empleado para ejecutar los patios interiores procede de las propias excavaciones realizadas durante la ejecución de las obras, y al tratarse de un suelo arcilloso con carácter expansivo, éste ha podido cambiar de volumen como consecuencia de la modificación de su proporción natural de humedad, o tras ser remoldeado para su empleo como relleno, deformación que ha favorecido la rotura de uniones en la red de saneamiento.

Debido a que el forjado sanitario conecta longitudinalmente las seis comunidades que forman la manzana, las filtraciones de agua pueden proceder de la red del edificio en el que intervenimos o de la instalación de los edificios colindantes.

\subsubsection{Retroceso desde la red de alcantarillado}

Se comprueba que en época de fuertes lluvias se produce el retroceso de agua desde la red pública de saneamiento como consecuencia de la falta de sección de la instalación y de su falta de mantenimiento, hecho constatado por el continuo atasco de imbornales y pozos de acometida.

El retroceso de agua provoca la puesta en carga de la red privativa del edificio, que permite su filtración de agua hacia el forjado sanitario desde las arquetas de paso previas a la sifónica.

\subsubsection{Agua pluvial filtrada desde el encuentro del acerado y la fachada}

Se evidencia que el acerado de fachada trasera se encuentra agrietado y levantado como consecuencia del empuje de las raíces de árboles y arbustos ubicados en las zonas ajardinadas intercomunitarias.

Por otra parte se observa la existencia de juntas entre el acerado y las fachadas que permiten la filtración de agua pluvial y de riego hacia el forjado sanitario, así como agua pluvial recogida por los bajantes ubicados en fachada, y que evacuan el agua hacia el acerado.

\subsubsection{Agua pluvial o de riego filtrada desde las zonas ajardinadas}

El agua de riego y pluvial recogida por las zonas ajardinadas se filtra hacia el terreno, que por capilaridad puede circular hasta el forjado sanitario.

\subsection{Causa de los daños por grietas}

Como consecuencia del embalsamiento de agua se ha producido la saturación del terreno, que bajo las altas presiones transmitidas por los pozos de cimentación ha dado lugar a su asiento diferencial.

Generalmente dicho asiento se produce en la fachada delantera y trasera del edificio, provocando la rotura del encuentro más débil de la albañilería, correspondiente a la unión de la caja de escalera con la fachada interior de las viviendas ${ }^{2}$, según podemos deducir de la nivelación topográfica de la Figura 10.

Por otra parte comprobamos que como consecuencia de este asiento diferencial, y gracias a la rigidez del conjunto cimentación estructura ${ }^{3}$, se ha producido un giro simétrico en el edificio que ha permitido minimizar las distorsiones angulares de las estructura, reduciendo la aparición de los daños a los elementos descritos de zonas comunes.

Por último, realizada una cala para inspeccionar la sección y armado de los nervios de borde correspondientes a la caja de escalera del edificio C/ Ciudad de Onteniente 4, se comprueba que las piezas en $\mathrm{U}$ de hormigón prefabricado carecen de armadura y resultan estructuralmente independiente del nervio de borde reflejado en la Figura 11.

\footnotetext{
${ }^{2}$ Maximiliano Astroza y Andrés Schmidt (4) estudian la capacidad de deformación de muros de albañilería ante distorsiones angulares, determinado la tensión a partir de la cual se produce la fisuración del elemento.

${ }^{3}$ La tesis doctoral realizada realizada por $\mathrm{M}^{\mathrm{a}}$ del Pilar Rodríguez-Monteverde y Cantarll (5) determina el coeficiente de rigidez suelo-estructura y su contribución a la redistribución de cargas por deformación.
} 


\subsection{Causa de los daños por humedad capilar}

La falta de ventilación del forjado sanitario como consecuencia de la sectorización de los paños de forjado por las vigas de canto, así como la insuficiente sección de los huecos de ventilación del recinto, provoca la condensación del agua embalsada, lo que permite la humectación del forjado y la transmisión por capilaridad de la humedad hacia la solería y paramentos de la planta baja.

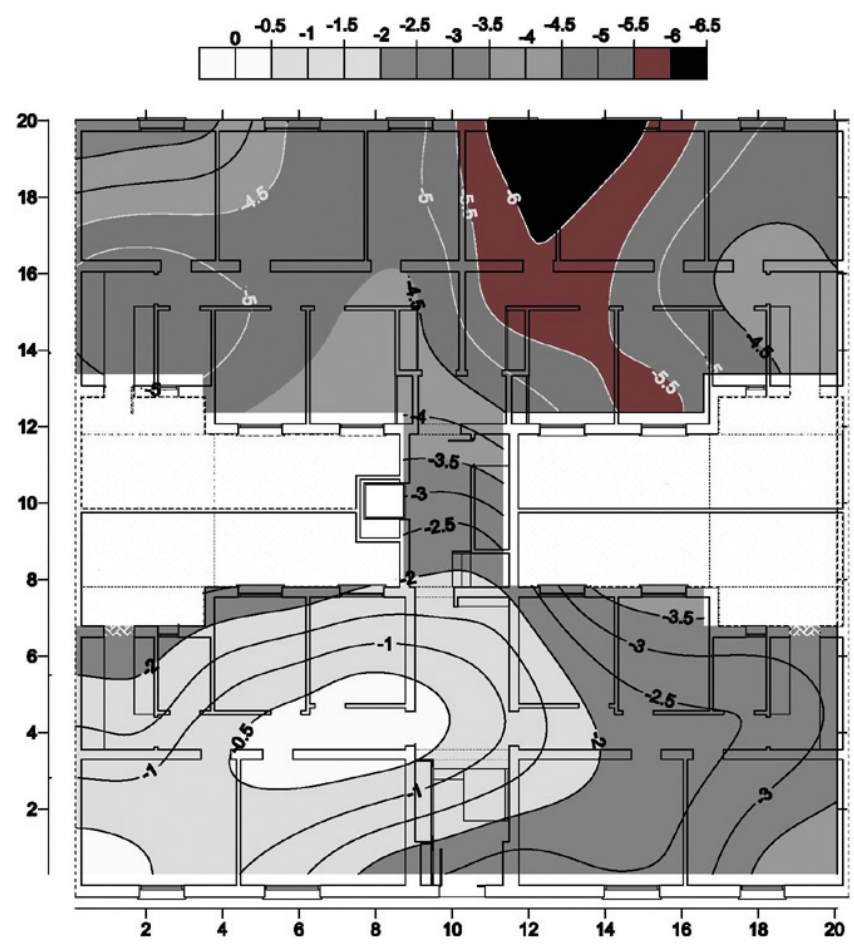

Figura 10. Nivelación topográfica del edificio sito en C/ Ciudad de Onteniente 4

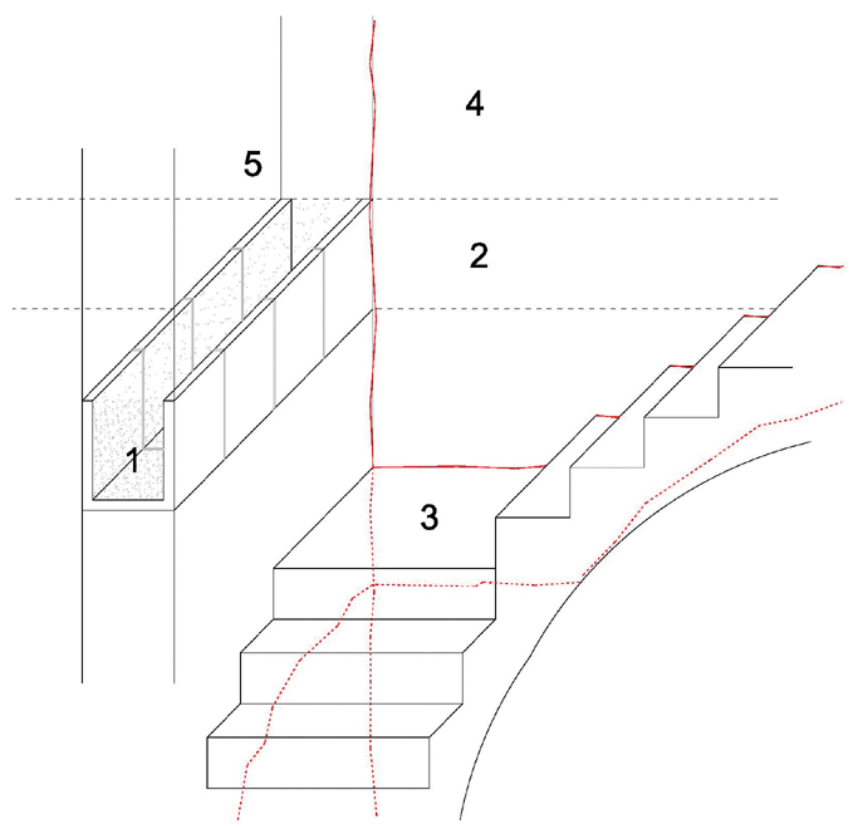

Figura 11. Axonometría de escalera. Correspondencia: 1. Nervio de borde sin armar; 2 Jácena de HA; 3 . Zanca de escalera a salta caballo; 4 y 5 muros de carga y cerramiento de bloques de hormigón vibrado.

\section{OBJETIVOS DE LA INTERVENCIÓN}

Tras verificar que no existe en ningún caso asientos diferenciales o distorsiones angulares que puedan poner en riesgo la estabilidad de la estructura según los datos obtenidos mediante la nivelación topográfica, y que las afecciones por grietas en cajas de escalera únicamente tienen mayor trascendencia en la comunidad de propietarios C/ Ciudad de Onteniente 4, se plantea una propuesta de intervención de carácter preventivo que responde a los siguientes objetivos:

1. Registrar el forjado sanitario para la observación y mantenimiento periódico de las redes de saneamiento y cimentación del edificio.

2. Facilitar la ventilación del forjado sanitario con objeto de eliminar la condensación de agua y por consiguiente la humedad capilar de planta baja.

3. Evacuar la posible filtración de agua con origen en causas en las que no podemos intervenir, tales como el agua procedente de redes de saneamiento colindantes, agua pluvial o de riego de zonas ajardinadas, o retroceso de agua desde la red de alcantarillado público.

4. Estabilizar la presencia de agua en las arcillas con objeto de limitar posibles cambios de humedad y por tanto el aumento de volumen o la retracción del terreno.

5. Cortar el paso a las raíces de los árboles ubicados en las zonas ajardinadas.

6. Sustituir la red de saneamiento de la comunidad en la que se interviene.

7. Sustitución del acerado trasero y el sellado de todo el perímetro en su encuentro con el cerramiento.

\section{PROPUESTA DE INTERVENCIÓN}

Para cumplir con los objetivos 1 a 6, se plantea la ejecución de calles de ventilación excavadas en el terreno que cruzan longitudinal y transversalmente el edificio, y que permite una ventilación cruzada mediante la disposición de cuatro huecos de ventilación en fachada delantera y trasera, y la disposición de un conductor de extracción en cada patio interior, según Figura 12.

Estas calles tienen una dimensión de 1 metro de profundidad y 1 metro de ancho. Constan en su base de un film de polietileno sobre el que se ejecuta una capa de hormigón en masa con una formación de pendiente del 1\%. En la generatriz de la calle se dispone una red drenante de recogida de aguas de $\varnothing$ $160 \mathrm{~mm}$ cubierta con un geotextil y una capa de gravas de 15 cm, según muestra la Figura 13.

Esta red drenante se conecta mediante una arqueta de bombeo a la red de saneamiento, permitiendo la evacuación del agua filtrada independientemente de su procedencia, según la Figura 14.

Para garantizar la estabilización de la presencia de agua en el terreno y la contención del terreno excavado, se dispone una malla de gallinero hexagonal de 1,5 cm anclada mediante tochos en la cabeza del talud, según se extrae de la Figura 15 .

Una vez ejecutadas las calles se procede a la sustitución de la red de saneamiento existente, por una instalación flexible de PVC colgada del forjado sanitario para evitar su apoyo en el terreno. 


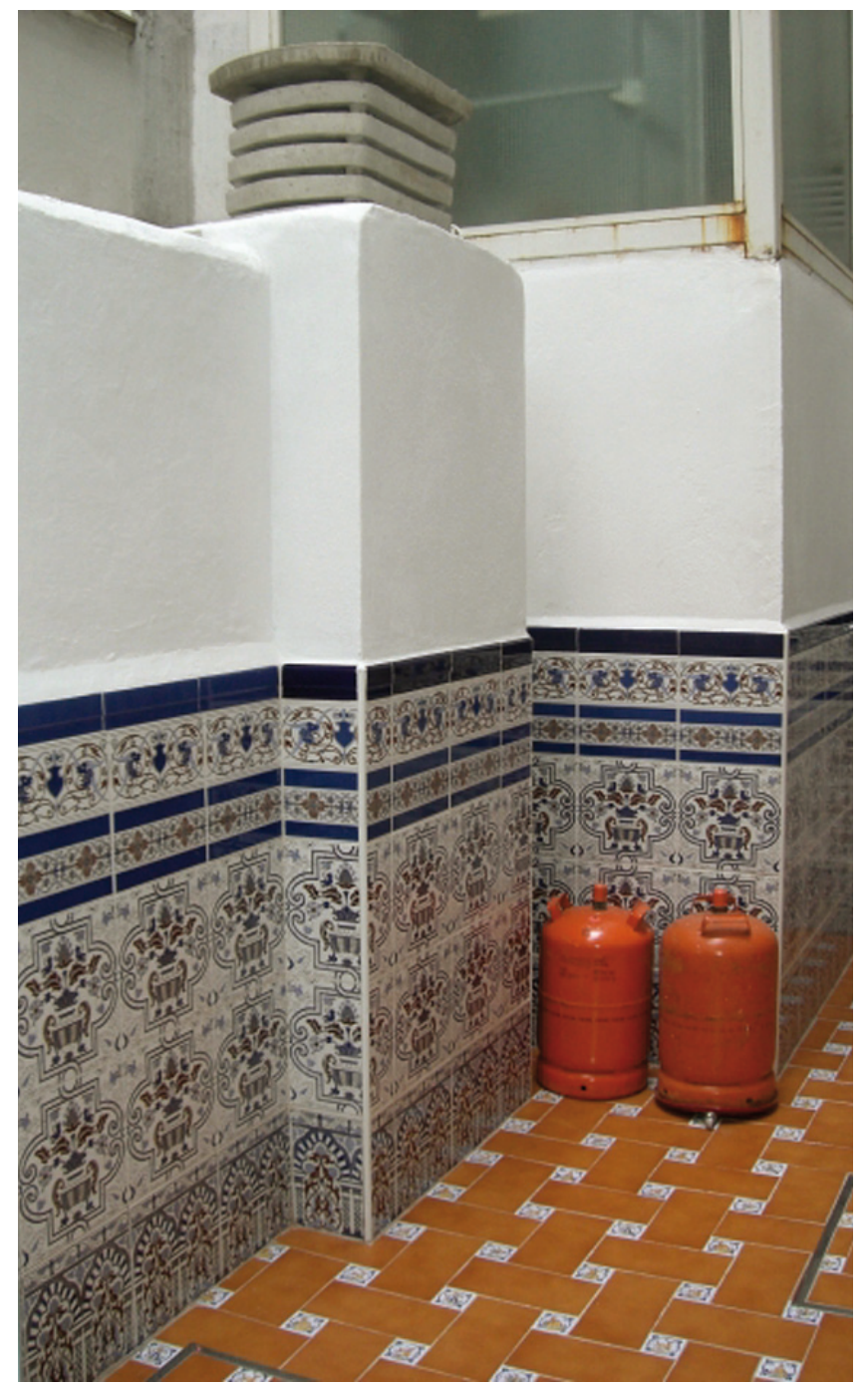

Figura 12. Conducto de extracción ubicado en el patio interior intercomunitario.

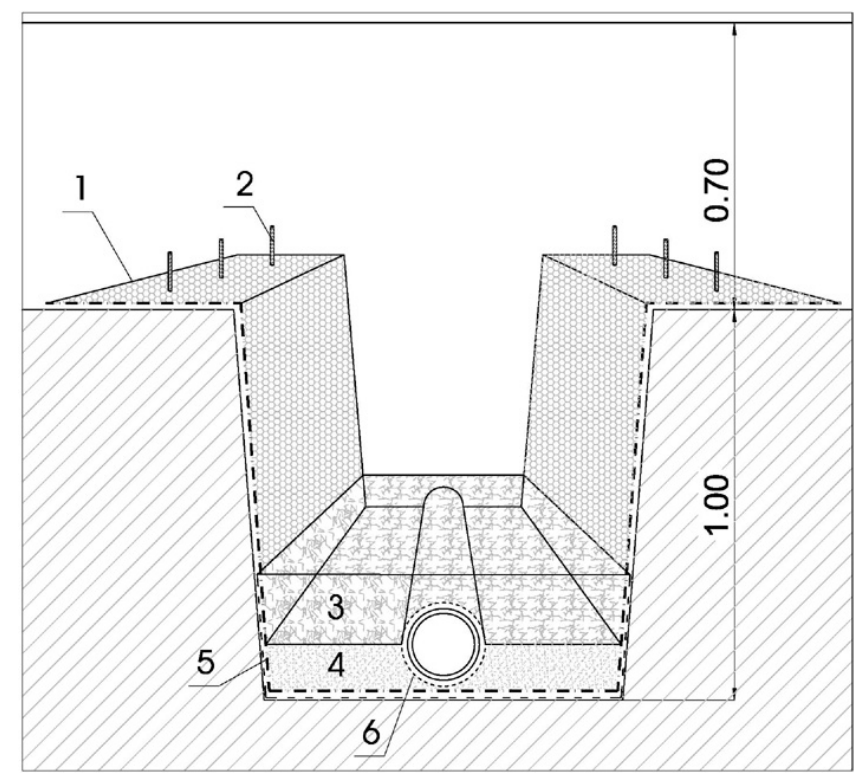

Figura 13. Detalle constructivo de calle de ventilación. Correspondencia: 1) malla metálica exagonal de 1,5 cm de espesor. 2) Ø 12 cada $50 \mathrm{~cm} .3$ ) Capa de grava de $15 \mathrm{~cm}$ de espesor. 4) Capa de hormigón en masa de $15 \mathrm{~cm}$ de espesor. 5) Film de polietileno. 6) Tubo drenante de diámetro $160 \mathrm{~mm}$.
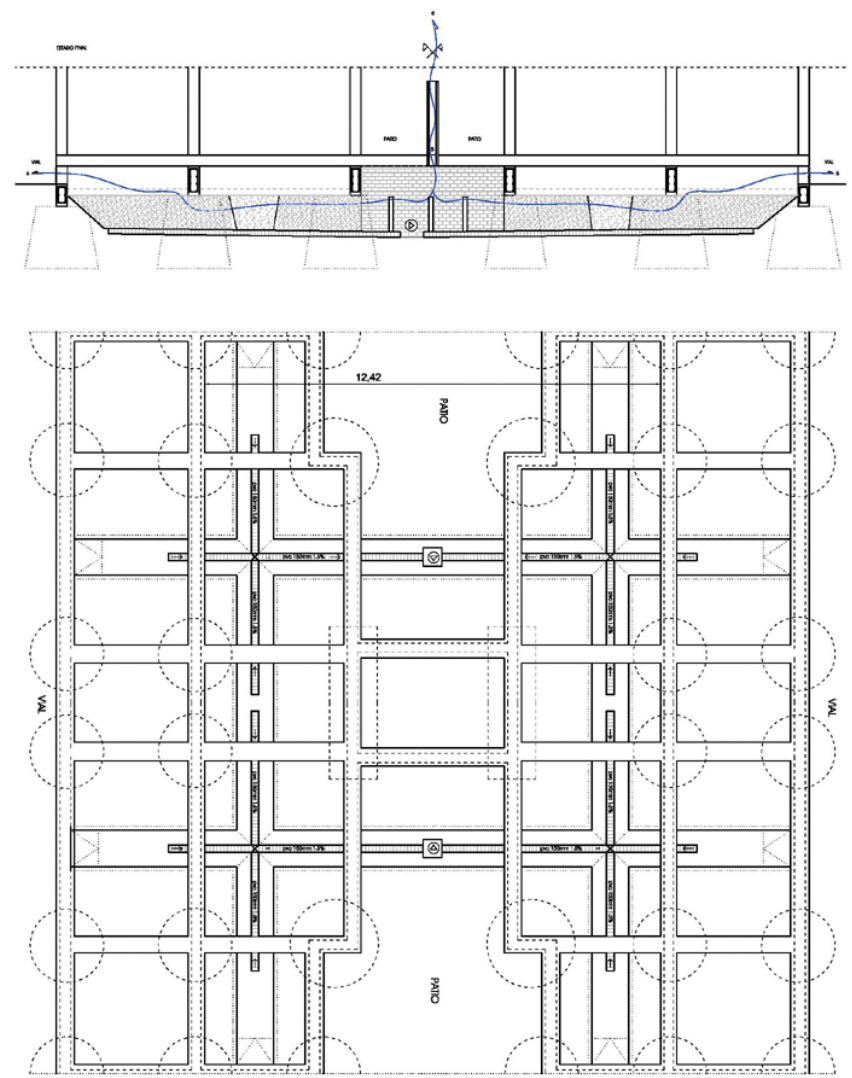

Figura 14. Esquema en planta de calles ventilación y red drenante.

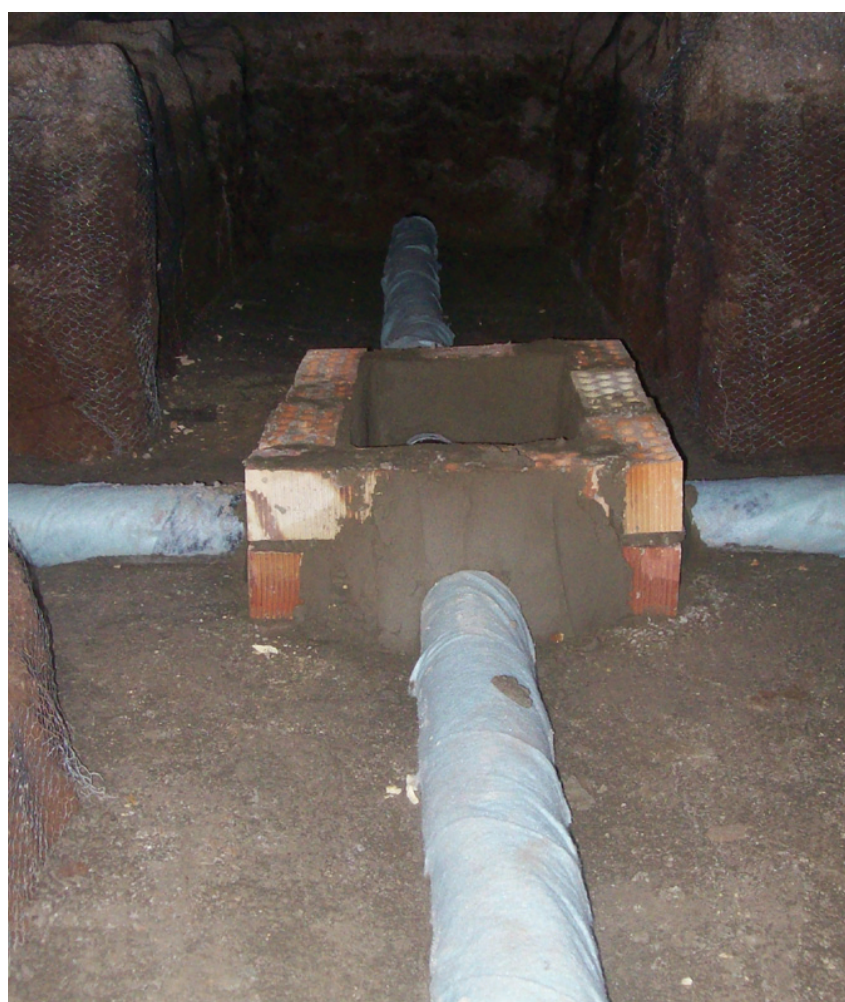

Figura 15. Disposición de red drenante

El acceso al forjado sanitario se realiza desde los patios interiores mediante arquetas de registro, lo que requiere la ejecución de una excavación entibada y la creación de un recinto donde los propios codales de fábrica de ladrillo configuran la arquea de bombeo. 
Esta intervención se llevará a cabo posteriormente en las comunidades colindantes hasta colmatar la totalidad de la manzana, lo que permitirá el registro de todas las instalaciones de saneamiento y el forjado sanitario completo desde cualquiera de las comunidades de propietarios según Figura 16.

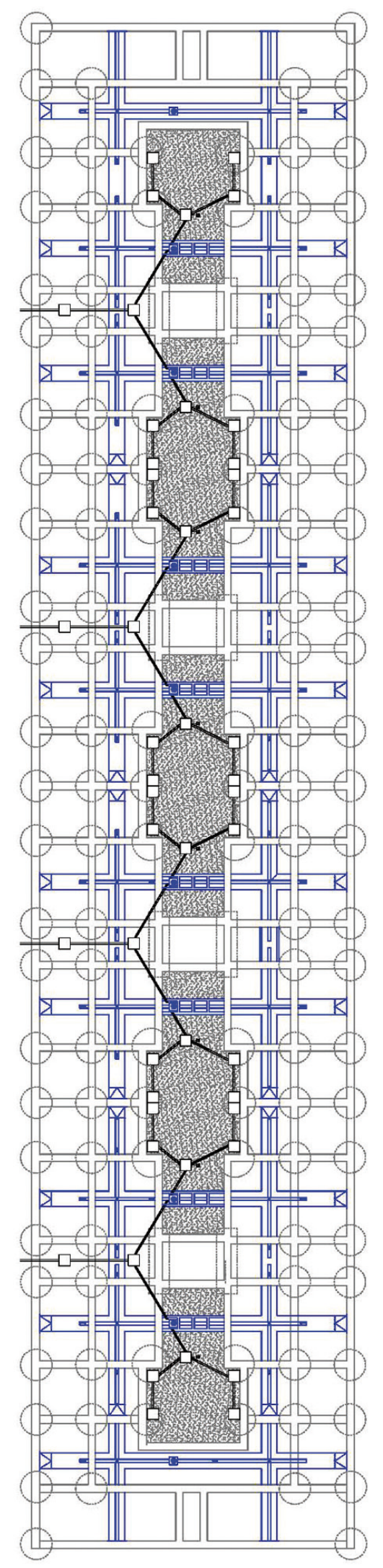

Figura 16. Calles de ventilación en manzana.

\section{CÁLCULOS DE LA VENTILACIÓN}

El Código Técnico de la Edificación, Documento Básico de Salubridad (6), no establece la ventilación mínima exigida para un forjado sanitario al no tratarse de una zona habitable. Por ello se toma como referencia el caudal de ventilación mínimo exigido para un núcleo húmedo, siendo aproximadamente $q_{v}=2,00 \mathrm{l} / \mathrm{s}$ por $\mathrm{m}^{2}$ de superficie.

Contamos con una superficie en planta de 404,00 $\mathrm{m}^{2}$ por comunidad, con lo que el caudal necesario será $q_{v}=808,00 \mathrm{l} / \mathrm{s}$.

Los huecos de ventilación abiertos a fachada o aberturas de admisión, así como el conducto de ventilación o abertura de extracción, deben garantizar respectivamente un área efectiva según la tabla 4.1 del Documento Básico $A=4 \cdot q_{v} \mathrm{~cm}^{2}$, es $\operatorname{decir} A=3.232,00 \mathrm{~cm}^{2}$.

Se disponen 4 rejillas de ventilación en fachada delantera y 4 en fachada trasera con un área efectiva de $30 \times 60 \mathrm{~cm}^{2}$, lo que hace un total de $14.400,00 \mathrm{~cm}^{2}$, superior a la requerida.

Por otra parte se disponen dos conductos de ventilación, uno por patio, con un área efectiva de $80 \times 30 \mathrm{~cm}^{2}$, lo que hace un total de $4.800,00 \mathrm{~cm}^{2}$, superior a la requerida.

\section{COSTE ECONÓMICO}

El presupuesto de ejecución material de los tres capítulos de obra que engloban la intervención, calles de ventilación, sustitución de red de saneamiento, e intervención en acerados ascendió para los dos modelos edificatorios existentes en la manzana a las siguientes cantidades:

Tipo A.

- Red de saneamiento $\mathrm{H}$

$5 \cdot 719,69 €$

- Calles de ventilación

$8.403,13 €$

- Acerados

Total PEM

$1.715,17 €$

Total PEC

$15.837,99 €$

$22.239,70 €$

Tipo B.

- Red de saneamiento H 10.399,44€

- Calles de ventilación 21.007,84€

- Acerados $\underline{2.731,20 €}$

Total PEM $\quad 34.138,48 €$

Total PEC $\quad 47.937,25 €$

El coste total de la intervención en el bloque aislado ascendería a $236.228,41 €$ de presupuesto de contrata, considerando 2 comunidades tipo A ubicadas en los extremos y 4 comunidades tipo B, suponiendo un coste por vivienda de 1.968,57€.

\section{CONCLUSIONES}

Los daños constructivos descritos surgen como consecuencia del embalsamiento de agua en la cámara bajo el forjado sanitario, lo que ha dado lugar a asientos diferenciales en la cimentación y humedad por condensación en la planta baja de los edificios.

Este embalsamiento se produce por una concurrencia de causas que no siempre están relacionadas con patologías procedentes del edificio en el que intervenimos. 
Por otra parte los daños surgidos como consecuencia de asientos diferenciales no han puesto en riesgo la estabilidad del edificio, cuya cimentación y estructura están bien diseñadas y dimensionadas, aportando una rigidez al conjunto edificado que ha evitado la aparición de distorsiones angulares no admisibles.

La intervención descrita se basa por tanto en plantear una actuación de carácter preventivo diseñada con el objetivo de erradicar la causa de las patologías, independientemente de su procedencia.
Transcurridos varios años de la terminación de las obras de rehabilitación en las comunidades descritas, se comprobó que de forma progresiva desaparecieron las humedades capilares en planta baja y los movimientos diferenciales se habían estabilizado, así como el espesor de las grietas existentes en la caja de escalera, por lo que se puede concluir que la solución técnica estudiada a permitido solventar a un bajo costo una patología constructiva, que en caso de agravarse podría plantear serios problemas para la estabilidad estructural del edificio y su habitabilidad.

\section{REFERENCIAS}

(1) Huete-Fuertes, R., Rodríguez-Liñán, C., Jaramillo-Morilla, A. (2005). Protocolo de Inspección Técnica de Edificios ITE. Sevilla: Colegio Oficial de Arquitectos de Sevilla.

(2) IGME. Mapa Geológico de España y Mapa Geológico General. Hoja 984/12-40. Instituto Geológico y Minero de España.

(3) IGME. (1986). Guía de previsión de riesgos por expansividad de arcillas. Instituto Geológico y Minero de España.

(4) Astroza, M., Schmidt, A. (2004). Capacidad de deformación de muros de albañilería confinada para distintos Niveles de desempeño. Revista de Ingeniería Sísmica, (70): 59-75.

(5) Rodríguez-Monteverde Cantarell, P. (2003). Influencia de las deformaciones diferidas del terreno sobre la redistribución de cargas y la fisuración de estructuras (Tesis doctoral). Madrid: Universidad Politécnica de Madrid.

(6) Código Técnico de la Edificación. (2009). Documento Básico de Salubridad CTE-DB-HS. http://www.codigotecnico. org/cte/export/sites/default/web/galerias/archivos/DB_HS_2009.pdf. 